\title{
Gender differences of basketball players aged 12-13 years according to the response to a moving object
}

\author{
Frolova L.S. ${ }^{* A B C D E}$, Kovalenko S.O. ${ }^{A D}$, Petrenko Yu.O. ${ }^{\mathrm{AD}}$, Tymofeev A.A. ${ }^{\mathrm{BCD}}$, Gunko P.M. ${ }^{\mathrm{BC}}$, Khomenko I.M. ${ }^{\mathrm{BC}}$, \\ Atamas O.A. ${ }^{\mathrm{AB}}$, Nechyporenko L.A. ${ }^{\mathrm{BE}}$, Nechyporenko D.L. ${ }^{\mathrm{BE}}$ \\ The Bohdan Khmelnytsky National University of Cherkasy, Ukraine
}

Authors' Contribution: A - Study design; B - Data collection; C - Statistical analysis; D - Manuscript Preparation; E - Funds Collection

\begin{abstract}
Purpose: $\quad$ The study aims at the determining gender differences of basketball players aged 12-13 years in response to an object in motion that determines the predominance of excitation and inhibition processes.

Material: $\quad$ The participants of the study are 58 basketball players aged 12-13 years, 35 are male basketball players and 23 are female. Male basketball players had 4-5-year training experience, female had 1,5-2-year experience. All athletes had normal visual acuity and were healthy at the time of the study. Young basketball players were informed about the purpose of the study. The computer program containing visual stimuli, moving with acceleration from different points of the monitor was used for testing.

Results: $\quad$ The deviations from the target data as well the pole were taken into consideration. It demonstrated the predominance of excitation and inhibition processes of basketball players of different sexes. The response accuracy to visual stimuli without taking into account the pole during perception with the left eye and the right eye is shown.

Conclusions: It was found out that the females' response accuracy during monocular perception is more closely related to the response accuracy during binocular imaging, whereas the role of the males' leading eye is increased during perception of objects from a convenient side. This may indicate the peculiar reactions under the conditions of double perception of objects in solving complex spatial problems.

Keywords: spatial, response, accuracy, binocular, monocular, perception, excitation, inhibition.
\end{abstract}

\section{Introduction}

Reactions to a moving object refer to sensomotor functions of a person, which are measured by the accuracy of space-timemotion characteristics [1]. Such characteristics in sports activities affect the motor abilities of athletes [2] as well as their cognitive abilities. Therefore, the use of the reaction to a moving object in sports is a widespread scientific practice. Performing the corresponding motion on a specific signal (visible spatial combination of two or more objects in motion) [1] testifies to the effectiveness of motor activity of athletes [3].

The response accuracy indicators point to the individual differences in the sensomotor response accuracy, as well as the predominance of excitation and inhibition processes [4]. The reaction to a moving object testing (RMO) can be carried out using various modern techniques. The most popular computer programs are the following: "Diagnostician-1" [4], "Multipsychometer-05" [5] and "The Pointer Indicator" [2], which include or are the tests of the RMO determination. These techniques provide the direct or circle movement of the object to the mark in one direction with a given speed, and the indicator is the response time and the response accuracy [1].

"The Sniper" [6] technique differs from the abovementioned. The object moves from different starting positions on the screen and accelerates, approaching the mark. In this test, the response accuracy from the center of the mark is measured in millimetres. Despite the fact that

(C) Frolova L.S., Kovalenko S.O., Petrenko Yu.O., Tymofeev A.A., Gunko P.M., Khomenko I.M., Atamas O.A., Nechyporenko L.A., Nechyporenko D.L., 2018

doi:10.15561/18189172.2018.0505 the forms of the test, the number of presentations, stimuli parameters and the criteria of the reaction evaluating are different, they have the same principle of action the response accuracy to a specific signal. Such a wide range of the RMO test programs indicates the modern technological capabilities of scientific search for precise diagnostics of sensomotor reactions of athletes. These techniques allowed carrying out a number of studies, the results of which demonstrate a scientific interest in the problem.

The areas of the research were the following: the response to a moving object in the complex study of psychophysiological functions of athletes of all ages [4], elite athlete [5], qualifications [7], and sexes [8], high class athletes [9]; the use of response to a moving object for sports specialization [10], the Impact of a sports vision training program [11], for technical and tactical training [12]; development of the testing technique and assessment of the sensomotor response accuracy [13].

The response to a moving object is an important criterion for assessment of the athletes' activity of games-based sports [14]. In games-based sports, a stable accuracy of the reaction to the position change, direction and speed of objects are required from an athlete in various situations.

The probability of error in responding to a moving object can lead to a technical fail of players $[2,3]$.

The response accuracy to a moving object in the complex of neurodynamic functions characterizes the individual and typological characteristics of athletes [7].

The sensomotor reactivity features, along with 
other psycho-physiological functions, are the effective basis of the sports activity of the games-based sports representatives $[15,16]$. Nowadays, the study on the athletes' response accuracy considering the binocular and monocular perception has not been presented yet. Spacetime information on approaching of an object is important for coordinating the components of the musculoskeletal system of athletes [17], which affects the accuracy and speed of their response [18].

The reaction to a moving object is used as one of the criteria for the nervous processes balance [1].

However, the predominance of excitation and inhibition processes data essential for young basketball players of both sexes in the account of binocular and monocular perception was not found out.

Purpose: The study aims at the determining gender differences of basketball players aged 12-13 in response to an object in motion that determines the predominance of excitation and inhibition processes.

\section{Materials \& methods}

\section{Participants}

The participants of the study are 58 basketball players aged 12-13 years, 35 are male basketball players and 23 are female. Male basketball players had 4-5-year training experience, female had 1,5-2-year experience. All athletes had normal visual acuity and were healthy at the time of the study. The study protocol was approved by the Ethical Committee of University. In addition, the children and their parents or legal guardians were fully informed about all the features of the study, and a signed informedconsent document was obtained from all the parents.

\section{Instruments}

The reaction to a moving object testing was carried out using the computer program "Sniper" [6]. In the center of the screen there is a circle (a target) and several similar targets around its periphery (tools). The background of the screen is white, which is neither distracting nor distracting. Fixed stimulus (central circle) is red in order to attract attention as a target. Moving stimuli (peripheral circles) are green and potentially safe during the multiple perceptions. The diameter of the target and the tool, the motion speed and the tool acceleration can be regulated, depending on age, gender, qualifications or specialization. The informative parameters for the games-based sports athletes aged 12-16 were determined experimentally: the diameter of the target and the tool $-10 \mathrm{~mm}$, the tool speed - $200 \mathrm{~mm} / \mathrm{s}$, acceleration to $100 \mathrm{~mm} / \mathrm{s}$, the number of moving stimuli -30 .

The function based on the number of accurate responses to the peripheral circles moving right and left on the monitor is also included.

\section{Procedure}

Testing of the participants was carried out in a day off from training. The participant settled comfortably in front of the computer, so that he could clearly see the monitor.

After pressing the start key, one of the peripheral circles (the tool) began to move to the central circle (the target) randomly. The participant must press the key at the moment of the possible coincidence of the central and peripheral circles centres.

The release of the key determined the beginning of the movement of the next peripheral circle. Pressing the key during the response was performed by a dominant hand. A non-dominant hand was holding a blind that covered one eye during monocular perception. The participant's right and left eye binocular and monocular perception was tested. After completing the presentation of 30 movable stimuli of each type of perception the testing was stopped and indicators were displayed.

\section{Analysis of sensomotor response data}

During the testing of each participant, the distance between centers of peripheral and central circles was recorded in millimeters [19]. The average distance and median were calculated automatically. The similar indicators were determined for the right and the left sides of the peripheral circle movement during the perception of stimuli with both eyes as well as with the left and the right eye separately.

The distance specified may be negative in reactions to the center of the central circle and positive while passing it. The relevance between the nervous processes in condition of their unbalance was performed considering the relative frequency of exact responses. The negative median over $10 \mathrm{~mm}$ indicates the predominance of excitation processes. The positive median of more than 10 $\mathrm{mm}$ indicates the predominance of inhibition processes. The median up to $1 \mathrm{~cm}$, regardless of the pole, described the relatively balanced nervous system.

\section{Statistical Analysis.}

The results of the research were processed using the traditional mathematical statistics program Excel. The arithmetic average and standard deviation of the average values of the distance between centers of stimuli without taking into account the pole were calculated for each group of participants. The median was calculated considering the deviation pole. Differences in the groups of participants indicators were considered to be correct at the point value $\mathrm{P}<0,05$.

\section{Results}

The reaction of young basketball players of different sexes to a moving object during binocular perception was studied (Table 1).

According to the indicators of reaction to objects moving from the left zone, male basketball players showed a predominance of excitation processes $(\mathrm{Me}=-1,69 \mathrm{~cm})$. General indicators and indicators of reaction to objects moving from the right zone indicated the balance of the nervous system of male basketball players $(\mathrm{Me}=-0,80$ $\mathrm{cm} ; \mathrm{Me}=-0,58 \mathrm{~cm})$. The indicators of reaction to objects moving from the right zone of female basketball players demonstrated the inhibition processes predominance $(\mathrm{Me}$ $=2,01 \mathrm{~cm})$. General indicators and indicators of reaction to objects moving from the left zone indicated the balance of the nervous system of females $(\mathrm{Me}=0,81 \mathrm{~cm} ; \mathrm{Me}=$ $0,57 \mathrm{~cm}$ ). In this case, the response accuracy of young basketball players of different sexes during binocular 
Table 1. Reaction indicators of basketball players aged 12-13 years to a moving object during binocular perception.

\begin{tabular}{|c|c|c|c|c|}
\hline \multirow{2}{*}{ Indicators } & \multicolumn{2}{|c|}{ Male $(n=35)$} & \multicolumn{2}{|c|}{ Female $(n=23)$} \\
\hline & Me & $\bar{X} \pm S$ & Me & $\bar{X} \pm S$ \\
\hline General average distance of moving objects perception, $\mathrm{cm}$ & $-0,80$ & $2,00 \pm 0,28$ & 0,81 & $1,84 \pm 0,36$ \\
\hline $\begin{array}{l}\text { General average distance of objects perception, moving from } \\
\text { the left zone, } \mathrm{cm}\end{array}$ & $-1,69$ & $3,05 \pm 0,47$ & 0,57 & $2,75 \pm 0,54$ \\
\hline $\begin{array}{l}\text { General average distance of objects perception, moving from } \\
\text { the right zone, } \mathrm{cm}\end{array}$ & $-0,58$ & $2,43 \pm 0,31$ & 2,01 & $2,87 \pm 0,39$ \\
\hline
\end{tabular}

perception of moving objects was almost identical $(\mathrm{P}>0,05)$.

The reaction of the young basketball players of different sexes to a moving object during the left eye monocular perception (Table 2) was studied.

According to the indicators of the reaction to objects moving from the right zone, the results of male basketball players showed a predominance of excitation processes $(\mathrm{Me}=-1,13 \mathrm{~cm})$. Considering the indicators of the reaction to objects moving from the left zone as well as general indicators, the nervous system of male basketball players may be characterized as balanced $(\mathrm{Me}=-0,67$ $\mathrm{cm} ; \mathrm{Me}=0,03 \mathrm{~cm})$. According to general indicators and indicators of the reaction to objects moving from the left zone, the processes of inhibition $(\mathrm{Me}=1,37 \mathrm{~cm}, \mathrm{Me}=$ $1,66 \mathrm{~cm}$ ) predominated among female basketball players. During the females' perception of objects moving from the right zone, the balance of nervous processes was detected $(\mathrm{Me}=0,60 \mathrm{~cm})$. The response accuracy of male basketball players during the left eye perception was higher than that of the females according to the general indicators of motion of objects from different monitor zones by $0,86 \mathrm{~cm}(\mathrm{P}<0,05)$. The response accuracy during the perception of objects moving from the right and left zones was compared. The male basketball players showed higher response accuracy than female during the perception of objects moving from the left zone by 1,05 $\mathrm{cm}(\mathrm{P}<0,05)$.

When perceiving objects moving from the right zone, the indicators of the response accuracy of the males and females were on the same level $(\mathrm{P}>0,05)$.

The reaction of the young basketball players of different sexes to a moving object during the right eye monocular perception (Table 3) was studied.

According to the general indicators and indicators of the reaction to objects moving from the right zone, male basketball players demonstrated the balance of the nervous processes $(\mathrm{Me}=-0,17 \mathrm{~cm} ; \mathrm{Me}=0,40 \mathrm{~cm})$. According to the indicators of the reaction to objects moving from the left zone, the inhibition processes prevailed $(\mathrm{Me}=-1,08$ $\mathrm{cm})$. According to general indicators and indicators of the females' reaction to objects moving from the right zone,

Table 2. Indicators of the reaction to a moving object of basketball players aged 12-13 aged during monocular perception (the left eye).

\begin{tabular}{lllll}
\hline \multirow{2}{*}{ Indicators } & \multicolumn{2}{l}{ Male (n=35) } & \multicolumn{2}{l}{ Female (n=23) } \\
& Me & $\bar{X} \pm S$ & Me & $\bar{X} \pm S$ \\
\hline General average distance of moving objects perception, $\mathrm{cm}$. & $-0,67$ & $1,35 \pm 0,21^{*}$ & 1,37 & $2,21 \pm 0,43$ \\
$\begin{array}{l}\text { General average distance of objects perception, moving from the } \\
\text { left zone, cm. }\end{array}$ & 0,03 & $1,81 \pm 0,28^{*}$ & 1,66 & $2,86 \pm 0,47$ \\
$\begin{array}{l}\text { General average distance of objects perception, moving from the } \\
\text { right zone, } \mathrm{cm} \text {. }\end{array}$ & $-1,13$ & $2,20 \pm 0,33$ & 0,60 & $2,46 \pm 0,52$ \\
\hline
\end{tabular}

Note: ${ }^{*} p<0,05-$ in comparison with female basketball players

Table 3. Indicators of the reaction to a moving object of basketball players aged 12-13 years during the monocular perception (the right eye).

\begin{tabular}{|c|c|c|c|c|}
\hline \multirow[b]{2}{*}{ Indicators } & \multicolumn{2}{|c|}{ Male $(n=35)$} & \multicolumn{2}{|c|}{ Female $(n=23)$} \\
\hline & Me & $\bar{X} \pm S$ & Me & $\bar{X} \pm S$ \\
\hline General average distance of moving objects perception, $\mathrm{cm}$. & $-0,17$ & $1,63 \pm 0,29 *$ & 1,23 & $2,36 \pm 0,30$ \\
\hline $\begin{array}{l}\text { General average distance of objects perception, moving from the } \\
\text { left zone, } \mathrm{cm} \text {. }\end{array}$ & $-1,08$ & $2,18 \pm 0,29$ & 0,12 & $2,89 \pm 0,49$ \\
\hline $\begin{array}{l}\text { General average distance of objects perception, moving from the } \\
\text { right zone, } \mathrm{cm} \text {. }\end{array}$ & 0,40 & $2,31 \pm 0,58^{*}$ & 3,53 & $3,88 \pm 0,47$ \\
\hline
\end{tabular}

Note: ${ }^{*} \mathrm{P}<0,05$ - in comparison with female basketball players 
the inhibition processes prevailed $(\mathrm{Me}=1,23 \mathrm{~cm} ; \mathrm{Me}=$ $3,53 \mathrm{~cm}$ ). During the perception of objects moving from the left zone, the relative balance of the females' nervous processes was detected $(\mathrm{Me}=0,12 \mathrm{~cm})$.

The male basketball players showed higher response accuracy than the female during the left eye perception of objects moving from different zones by $0,73 \mathrm{~cm}(\mathrm{P}$ $<0,05)$.

The response accuracy during the perception of objects moving from the right and left zones was compared. The male basketball players showed higher response accuracy than the female during the perception of objects moving from right zone, by $3,13 \mathrm{~cm}(\mathrm{P}<0,05)$. During the perception of objects moving from the left zone, the male response accuracy is higher than female by $0,96 \mathrm{~cm}$ $(\mathrm{P}>0,05)$.

\section{Discussion}

The main task of our research is to determine the gender differences of sensomotor reaction to visual moving stimuli as a component of the technical and tactical readiness of basketball players of different sexes. The study of the response to an object in motion considering gender features contains controversial data.

The obtained sensomotor response rates indicate the dominance of both female [20] and male basketball players $[21,22]$. These data indicate the dependence of the response accuracyon the age, sex and sport experience. We also have data on the age-related sensomotor response of young athletes [23], but they do not indicate gender specifics. In our research the innovative testing method of the response to a moving object "Sniper" was applied [6]. It has been developed taking into account the features of team sport games, which consist of the response peculiarities to objects moving from different directions and with acceleration in the process of competitive activities. The principle of methodology operation used in the research is based on a visible combination of two objects. This is a peculiar feature of the programs of this kind [1]. However, unlike other techniques, the "Sniper" program [19] defines an indicator of timely response as an exact coincidence of the target and the moving object. The degree of deviation from the mark and the direction of the reaction are considered.

The response speed is determined by most of the RMO techniques, but the authors underline the importance of the response accuracy as a criterion for a complex sensomotor reaction of athletes $[10,13]$, which may point to individual differences [1]. The necessity of sensomotor reactivity evaluation of the athletes aged 12-13 is confirmed by N. Makarenko et al. [4]. According to these data, there is an increase in the attention concentration of the athletes aged 12, which depends on the speed of perception and processing of information. The authors believe that "under the influence of physical activity, the nervous control of the reliability of sensomotor reactions increases"[4]. It characterizes the training of the sensomotor system [20]. It should be noted that the sports training of young basketball players aged $12-13$ is a stage in the formation of tactical readiness, the basis of which is the interaction with a number of moving objects [23]. According to the sensomotor response accuracy, the degree of difficulty of the athlete's tasks can be determined [24] and the effectiveness of motor actions can be anticipated [25].

Problems solving based on the athlete's motor activity is closely related to the motor response to incentives as well as recognition of their qualities [25]. One of such qualities is a color. In the innovative technique "Sniper", the stimulus (moving element) is red. It operates at a minimum frequency perceived by the human eye and is one of the three "main" colors in the RGB system [26], it attracts attention quickly, stimulates the retina and brings a clear retinal image [27]. The immovable mark color is defined as green. It has been defined as a tranquilize color, an additional color in the RGB system [26]. The choice of such a combination of colors is based on the principles of the relationship between colored energies. Red stimulates excitation and acts as an irritant, and green suppresses irritation [26]. The colors combination is considered to be a strong contrast between energy and tranquility [27], which, in our opinion, will not significantly affect the emotional state of athletes, minimizing the monotony of testing and guaranteeing the high results accuracy. The human sensory input peculiarities can restrict the passthrough function and power of information processing [17]. In sports, the timeliness of actions depends on the speed of information processing and spatial perception is essential [18].

The object motion prediction, especially under the condition of acceleration depends on the spatial estimation accuracy [28]. Each sport is characterized by the perception specificity expressed by the specific forms of the visual analyzer functions [29]. It depends on the eyes position that serves as a spatial index for the visual image [12].

Basketball players use binocular localization of the main objects during perception of game situations [30]. However, athletes' visual analyzers perceive other objects that move from the right and left sides of the player beyond the main focus [11]. In this case, the response accuracy to the spatial deviations of the objects will depend on the leading eye and the stability of the visual asymmetry [31].

It is known that focusing on several moving objects leads to a decrease in the concentration of attention that affects the perception and the subsequent sensomotor response [23, 32].

At the same time, it has been proved that there are two subsystems that take part in the perception of two moving objects, which contributes to the greater effectiveness of information processing [33]. The asymmetry of sensor systems is a children's characteristic feature [34], but under the influence of sports training, these features can acquire signs of symmetry, as there is adaptation to the certain type of mechanisms of spatial localization [29].

The results of our research also demonstrated symmetry of various types of spatial vision of basketball players aged 12-13 (the left and the right eye).

It means that during the 4-5 year-period of basketball 
playing, the role of the leading eye during the accented monocular perception of moving objects becomes less expressive due to the plastic nature and the lability of the interaction of monocular systems [29]. However, regardless of the environment perception with both eyes, the activity of basketball players is affected by sensory asymmetry. It is formed at an early age and lasts for a lifetime [35]. Studies have shown that the role of the leading eye of basketball players aged 12-13 was intensified in the process of changing of the visual analyzer to objects moving from different directions. The male basketball players had better perception of moving objects from the left zone (with both the right and the left eye), compared to binocular vision.

The female basketball players' right sensory input was less powerful during the perception of moving objects from the right zone, compared to the binocular and monocular (the left eye) perception. The response accuracy based on the visual asymmetry can characterize the effectiveness of the athlete's actions [24]. That is, the male basketball players while focusing on the main object with both eyes during the active movement of other objects from the left zone can increase the effectiveness of tactical thinking. The effectiveness of tactical thinking of the female basketball players at the moment of focusing on the main object with both eyes and active movement of other objects from the right zone may decrease.

Sensory asymmetry is the basis of tactical thinking [35], the main period of its formation is between the ages of 12 and 16 [16].

This emphasizes the importance of taking into account visual asymmetry as a criterion of neurodynamic peculiarity in the process of multi-year training of young athletes [35]. In our study gender differences in the spatial response accuracy were detected: during monocular perception of moving objects with the left eye from the left and with the right eye from the right. This response is related to the field of peripheral vision horizontally. The peripheral detection of the moving object is more accurate, compared to the detection of the shape, which depends on the characteristics of the visual analyzer [36].

It should be noted that the training experience expands the athletes' field of vision significantly. It contributes to a more accurate perception of the object and increases the speed of information processing [37]. With regard to gender differences, the females' peripheral vision is significantly better than males' [38]. Gender differences of female basketball players aged 12-13 years consists in the wider field of vision[36]. Our study shows, that male basketball players advantages of female basketball players are stipulated by their extensive basketball playing experience (4-5 years vs 1,5-2 years), which greatly affects the field of vision [39].

Probably, female basketball players' results were influenced not only by the speed of information processing, but also by the reduced ability to predict the space-time feature of the target trajectory of a moving object [23].

However, the improvement of the sensory motor response during long training process is common for both female and male basketball players [20, 22]. The vast majority of studies on a moving object response are represented by the time characteristics of the sensomotor reaction $[1,2]$, while in our research spatial parameters are being analyzed. The reaction to a moving object is a complex sensomotor skill, which is formed not only due to estimation of the motion speed [13], but also due to spatial accuracy [2]. During visual stimuli response, the athlete performs spatial tasks and demonstrates specific abilities in visual space-time orientation that contributes to successful sports specialization $[3,16]$. Therefore, regardless of the characteristics of the visualmotor function, it is possible to compare the reaction characteristics according to the exact parameters. The sensomotor functions formation of children especially their spatial and temporal characteristics are significantly affected by the peculiarities of the main nervous processes. Their role in the formation of complex sensomotor functions of children aged 12-13 is weakened [38]. However, the complexity of the visual-motor tasks during the training of young athletes affects the speed of perception and processing of information positively [4]. The authors did not analyze non-athletes' results aged 1213 , since there is a comparison of trained and untrained people results that points to the advantage of the sensorymotor response speed of people with sporting experience and their better efficiency in the use of visual information $[21,37]$. In addition, there is information on the positive effects of long-term physical activity on solving complex problems for the development of sensomotor and cognitive functions of athletes [37].

It is also important to understand that the achievement of a high level of space-time characteristics of sensory functions during the laying of technical and tactical readiness foundation (ages of 11 and 16) will affect the quality of the skills implementation in the future [30].

Moreover, the qualified athletes of the game-based sports are characterized by short latent periods of complex visual motor reactions [1,7] and high response accuracy to a moving object $[2,15]$. It refers especially to highly skilled attacking players and affects the result of the game [7]. Analyzing the sensomotor reaction data it is important to take into account the magnitude of the mark deviation. Such a necessity is considered to be significant for the team sports selection $[2,3]$ as well as for the purposeful formation of athletes' significant professional skills [29].

But the deviation rate also determines the degree of the visual analyzer formation [1]. During binocular perception, the visual analyzers are characterized as mature enough to respond effectively to stimuli in a sporting activity. However, gender differences during binocular perception response were not detected.

The results of our research cannot be compared to other studies, because there haven't been any studies concerning athletes aged 12-13 years.

According to Yongtawee \& Woo, the visual and spatial abilities of boys and girls aged 15-16 years did not differ in stimuli response [20]. Studies concerning the adults showed higher rates of male process information ability 
to visual stimuli [8]. But during the objects recognition under the condition of barrier perception the response accuracy demonstrated by females was higher [39]. The visual input laterality, as a separate component of the psychological organization of a person [34], determines the peculiarities of the bioelectric activity transformation of the cerebral cortex under the influence of the sport activity [31].

It emphasizes the importance of studying the sensomotor characteristics during monocular perception. Our studies showed the male response accuracy prevalence over female in terms of deviation from the mark during monocular perception: perception of objects moving from the left zone with the left eye and perception of objects moving from the right zone with the right. We believe that this phenomenon can be explained by the cognitive differences in the analysis of the perception of females and males [39]. Solving complex spatial problems, males receive data through the sensor input. Female perception depends on the previous knowledge used to interpret the data [39], so they show their cognitive function better [8]. However, the time characteristics of sensomotor response accuracy of males are higher [8]. At the same time, males have an advantage over females in solving spatial problems, although females are better at handling varied tasks verbally [40]. Females' indicators during monocular perception may also be influenced by the switching attention variability (it's higher than males) [41], which reduces the effectiveness of the perception of moving objects. The stability of the motor reaction is one of the criteria of the nervous processes balance [1].

According to the indicators of the deviation direction of responses of young basketball players aged 1213 gender features were revealed. Males' response demonstrated the nerve processes balance peculiarities, which, when perceiving the object from an inconvenient visibility distance, transformed into excitement. Females' nerve processes balance during binocular perception transformed to the predominance of inhibition processes during the perception of objects from a convenient visibility distance. In the studies by Khalfina et al. concerning tennis players aged 7-8 years the gender differences were also revealed. However, the data obtained are opposite: the excitation processes dominated among females, and the processes of inhibition prevailed among males [42]. This confirms the importance of taking into account the age of athletes, which depends significantly on the rate of excitability and inhibitory processes [1].
The influence of specialization on the nervous processes peculiarities should not be ignored [25, 29]. Predominance of excitation processes is peculiar for the adult games-based sport athletes [10], especially extroverts [9]. Although skilled basketball players have excitation and inhibition processes balance [43].

\section{Conclusion}

No gender differences of basketball players aged 1213 in the spatial response accuracy to a moving object during binocular perception, regardless of the direction of objects movement were determined, that indicates the visual analyzer maturity to visual stimuli respond during sports activities. Compared to male basketball players aged 12-13, females' response accuracy during monocular perception is more closely related to the response accuracy during binocular visualization. However, the role of the leading eye of young male basketball players increased during the perception from a convenient side. This may indicate the specific reaction during double perception of objects in solving complex spatial problems. It is assumed to use the reaction to a moving object data to obtain the spatial characteristics accuracy of the games-based sport athletes of all ages and sex, taking into account the latent period of the visual motor reaction. The effect of excitation and inhibition processes on the functional state of athletes, depending on the nature of the sport activity and the direction of the training process, taking into account the functional asymmetry is also of great importance.

\section{Funding statement}

The research has been conducted at the full expense of its authors within the framework of the research project "Theoretical and methodical principles of mobilizational readiness of athletes of various qualifications" (0116 u 003858) of the Bohdan Khmelnytsky National University of Cherkasy.

\section{Acknowledgements}

We thank the Basketball Club "Cherkasky Mavpy" for the participants of the study and their organization for testing.

\section{Conflict of interests}

The authors declare that there is no conflict of interests. 


\section{References}

1. Makarenko NV, Lizogub VS. Motor reaction steadyness as one of the criteria of the nervous processes balance. Actual problem of transport medicine. 2015; 4 (42-I):93-97. (in Russian)

2. Polevshchikov MM, Rozhentsov VV. Accuracy of motor reactions as a criterion for games-based sports selection. Lesgaft University Academic notes. 2013; 6 (100):103-108. doi:10.5930/issn.1994-4683.2013.06.100 (in Russian)

3. Polevshchikov MM, Palagina NI, Rozhentsov VV. Response to moving object as a qualification criterion for team sports. Theory and practice of physical culture. 2015; 10:23.

4. Makarenko MV, Ivaniura IO, Sheiko VI. Research of psychophysiological functions of pupils of middle school age during prolonged physical activity. Physiological journal. 2002; 48 (5):56-61. (in Ukrainian)

5. Korobeinykov GV, Korobeinykova LG, Rynok TM, Myshchenko VS. Stareiushchye osobennosty stressoustoichyvosty u эlytnыkh sportsmenov. Visnyk Cherkaskoho universytetu. Seriia: Biolohichni nauky. 2015; 2:128-133. (in Ukrainian)

6. Petrenko YO, Kovalenko SO, Frolova LS, Liubchenko KM, Tymofeev AA, Atamas O. A method of determining excitation and inhibition of the central nervous system. Ukraine patent 118142. 2017 Aug 1. (in Ukrainian)

7. Lizogub V, Suprunovych V, Pustovalov V, Hrechukha S. Neurodynamic functions of basketball players in different playing roles. Sports Bulletin of the Prydniprovya. 2016; 2:81-84. (in Ukrainian)

8. Korobeinikova LG. The manifestation of neurodynamic functions features of elite combat athletes of different sexes. Bulletin of the Taras Shevchenko National University of Kyiv. 2014; 3 (68):18-21. (in Ukrainian)

9. Fedorchuk S, Lysenko E. The nature of the response of highly qualified athletes to the moving object under the conditions of psycho-emotional stress. Sports Science of Ukraine. 2017; 3(79):47-54. (in Ukrainian)

10.Kovtun AO. Use of computer psychophysiological researches in the study of influence of sports specialization on the level of students' sensomotor reactions. Scientific and methodological bases of information technologies usage in the field of physical culture and sports. 2017; 1:53-57. (in Ukrainian)

11.Schwab S, Memmert D. The Impact of a Sports Vision Training Program in the Youth Field Hockey Players. Journal of Sports Science \& Medicine, 2012; 11 (4):624-631.

12.De Freitas J, Myers NE, Nobre AC. Tracking the changing feature of a moving object. Journal of Vision. 2016; 16 (3): 22:1-20. doi:10.1167/16.3.22

13.Familnikova NV, Polevshchikov MM, Rozhentsov VV. Reaction accuracy estimation of a person to a moving object. Modern science-based technologies. 2016; 2 (1):176-179. (in Russian)

14.Petrova TG, Khasanova NN, Grechishkina SS, Agirov AA. Analysis of the Influence of Sports Classes in the Section on the Functional State of the Nervous System of Students. Theory and practice of physical culture.2014; 11:12-16.

15. Vovkanych L, Dunets-Lesko A, Penchuk A, Kachmar P. Features of sensomotor reactions of athletes of various sports specializations. Physical activity, health and sports, 2015; 2 (20):17-26. (in Ukrainian)

16.Frolova L, Glazyrin I, Navaretsky D. Anatomic, functional and psychophysiological criteria of primary and basic sports selection in women's handball. Physical Education, Sports and Health Culture in Modern Society, 2008; 3:345-349. (in
Ukrainian)

17.Carrasco M. Visual attention: The past 25 years. Vision Research. 2011; 51:1484-1525. doi:10.1016/j. visres.2011.04.012

18.Williams AM, Davids K, Williams JG. Visual Perception and Action in Sport. London: New Fetter Lane; 2000.

19.Petrenko YO, Frolova LS. Information Technologies in the Human Nervous System Study. Scientific and methodological foundations of the use of information technologies in the field of physical culture and sports, 2017; 1:82-85. (in Ukrainian)

20. Yongtawee A, Woo M.-J. The Influence of Gender, Sports, and Training Experience on Cognitive Functions in Adolescent Athletes. Exercise Science. 2017; 26 (2):159-167. doi:10.15857/sex.2017.26.2.159

21.Balasubraman M, Sivapala K, Nishanthi V, Kinthusa S, Dilani M. Effect of Dual-Tasking in Visual and Auditory Simple Reaction Times. Indian J Physiol Pharmacol. 2015; 59 (2):194-198.

22.Notarnicola A, Maccagnano G, Pesce V, Tafuri S, Novielli G, Biagi M. Visual-spatial capacity: gender and sport differences in young volleyball and tennis athletes and non-athletes. BMC Research Notes. 2014; 7:57. doi:10.1186/1756-05007-57

23.Rothenberg-Cunningham A, Newell KM. Children's agerelated speed-accuracy strategies in intercepting moving targets in two dimensions. Res $Q$ Exerc Sport.2013; 84 (1):79-87.

24.Mishchenko VS, Korobeinikova LG, Korobeynikov GV. Psychophysiological state of highly skilled athletes with different levels of neurodynamic functions. The Bulletin of Cherkasy National University. 2017; 2: 45-53. (in Ukrainian)

25.Gurov MB, Kapilevich LV. Perception of movements features of athletes depending on the orientation of the training process. Bulletin of Siberian Medicine, 2013; 12 (2): 195-199. (in Russian)

26.Andrushko LM, Yasinsky VP. Influence of red on human psychosomatics. Scientific bulletin of Lviv State University of Internal Affairs, 2014; 1:212-223. (in Ukrainian)

27.Dontsova AY, Verbytska TV. Color perception peculiarities. Scientific notes of the Central Ukrainian National Technical University, 2010; 10 (2):165-167. (in Ukrainian)

28.Bennett SJ, Bengui N. Is Acceleration Used for Ocular Pursuit and Spatial Estimation during Prediction Motion? PLoS ONE. 2013; 8 (5): e63382. doi:10.1371/journal. pone. 0063382

29. Matova MA. Asymmetry and symmetry formation of visual perception in the process of human activity. Questions of psychology, 1980; 1:64-72. (in Russian)

30.Grushko AI, Bochaver KA, Kvitchasty AV, Kovalov AV, Kabanov DY, Konstantinova MV, Kasatkin VN. Motor reaction time diagnostics in various sports. Sports psychologist, 2016; 1 (40):82-87. (in Russian)

31.Fomina EV. Influence of the dominant side of the visual touch input on the dynamic rearrangements of cerebral interhemispheric asymmetry of the EEG spectral power. Omsk Scientific Bulletin, 2006; 1 (34):231-235. (in Russian)

32.Holcombe AO, Chen W.-Y. Exhausting Attentional Tracking Resources with a single fast-moving object. Cognition, 2012; 123(2):218-228. doi:10.1016/j.cognition.2011.10.003

33.Oksama L, Hyönä J. Position tracking and identity tracking are separate systems: Evidence from eye movements. Cognition, 2016; 146:393-409.

34.Galyuk NA. The phenomenon of asymmetry of a person's visual perception. Bulletin of the Tomsk State Pedagogical University, 2006; 2 (53):5-9. (in Russian)

35. Shynkaruk O, Ulan A. Modern views on the manifestation 
of the left-handed in sport. Physical Education, Sports and Health in the Modern Society, 2016; 3 (35):117-122. (in Ukrainian)

36. Vater C, Kredel R, Hossner E.-J. Detecting single-target changes in multiple object tracking: The case of peripheral vision. Atten Percept Psychophys, 2016; 78: 1004-1019. doi:10.3758/s13414-016-1078-7

37.Moreno FJ, Luis V, Salgado F, Garcia AA, Reina R. Visual behavior and perception of trajectories of moving objects with visual occlusion. Percept Mot Skills, 2005; 101 (1):1320. doi:10.2466/pms.101.1.13-20

38.Tkhorevskii VI. Physiology of a person. Moscow: Physical education, education and science; 2001. (in Russian)

39. McGivern RF, Adams B, Handa RJ, Pineda JA. Men and Women Exhibit a Differential Bias for Processing Movement versus Objects. PLoS ONE, 2012; 7 (3): e32238. doi:10.1371/ journal.pone. 0032238
40.Li R. Why do women see differently from the way men see it? A review of gender differences in cognition and sports. Journal of Sport and Health Science, 2014; 3:155-162. doi:10.1016/j.jshs.2014.03.012

41.Solianik R, Brazaitis M, Skurvydas A. Sex-related differences in attention and memory. Medicine, 2016; 52 (6): 372-377. doi:10.1016/j.medici.2016.11.007

42.Khalfina RR, Galimova AS, Danilov AV. Gender peculiarities of psycho-physiological state of young tennis players'. Bulletin of the Kemerovo State University, 2014; 1 (57): 1820. (in Russian)

43. Minhalov OG, Drehval IV. Analysis of the functional state of sensomotor reactions and major nervous processes of athletes playing sports. Bulletin of Biological and Medical Problems, 2017; 4-2 (140):268-270. (in Ukrainian)

\section{Information about the authors:}

Frolova L.S.; (Corresponding author); http://orcid.org/ 0000-0003-0763-7509; I-f2014@vu.cdu.edu.ua; The Bohdan Khmelnytsky National University of Cherkasy; 81 Shevchenko blvd., Cherkasy, 18031, Ukraine.

Kovalenko S.O.; http://orcid.org/0000-0002-4631-0464; kovstas@ukr.net; The Bohdan Khmelnytsky National University of Cherkasy; 81 Shevchenko blvd., Cherkasy, 18031, Ukraine.

Petrenko Yu.O.; http://orcid.org/ 0000-0002-6348-2110; Petrenko62@gmail.com; The Bohdan Khmelnytsky National University of Cherkasy; 81 Shevchenko blvd., Cherkasy, 18031, Ukraine.

Tymofeev A.A.; http://orcid.org/ 0000-0002-9851-0257; 13tim@i.ua; The Bohdan Khmelnytsky National University of Cherkasy; 81 Shevchenko blvd., Cherkasy, 18031, Ukraine.

Gunko P.M.; http://orcid.org/0000-0002-0609-8550; gunkopeter@bigmir.net; The Bohdan Khmelnytsky National University of Cherkasy; 81 Shevchenko blvd., Cherkasy, 18031, Ukraine.

Khomenko I.M.; http://orcid.org/ 0000-0002-1330-3604; tmfv2016@ukr.net; The Bohdan Khmelnytsky National University of Cherkasy; 81 Shevchenko blvd., Cherkasy, 18031, Ukraine.

Atamas O.A.; http://orcid.org/0000-0003-4711-5211; helga_atamas@i.ua; The Bohdan Khmelnytsky National University of Cherkasy; 81 Shevchenko blvd., Cherkasy, 18031, Ukraine.

Nechyporenko L.A.; http://orcid.org/0000-0002-7118-9870; nnifizkult2014@ukr.net; The Bohdan Khmelnytsky National University of Cherkasy; 81 Shevchenko blvd., Cherkasy, 18031, Ukraine.

Nechyporenko D.L.; http://orcid.org/0000-0002-6116-2551; dentandf@gmail.com; The Bohdan Khmelnytsky National University of Cherkasy; 81 Shevchenko blvd., Cherkasy, 18031, Ukraine.

Cite this article as: Frolova LS, Kovalenko SO, Petrenko YuO, Tymofeev AA, Gunko PM, Khomenko IM, Atamas OA, Nechyporenko LA, Nechyporenko DL. Gender differences of basketball players aged 12-13 years according to the response to a moving object. Pedagogics, psychology, medical-biological problems of physical training and sports, 2018;22(5):252-259. doi:10.15561/18189172.2018.0505

The electronic version of this article is the complete one and can be found online at: http://www.sportpedagogy.org.ua/index.php/PPS/issue/archive

This is an Open Access article distributed under the terms of the Creative Commons Attribution License, which permits unrestricted use, distribution, and reproduction in any medium, provided the original work is properly cited (http://creativecommons.org/licenses/by/4.0/deed.en).

Received: 18.08.2018

Accepted: 10.09.2018; Published: 30.09 .2018 